09

\title{
Генерация мягкого рентгеновского излучения в компактном лазере на свободных электронах с умножением гармоник
}

\author{
(C) К.В. Жуковский \\ Московский государственный университет им. М.В. Ломоносова, \\ 119991 Москва, Россия \\ e-mail: zhukovsk@physics.msu.ru
}

(Поступило в Редакцию 15 декабря 2017 г. В окончательной редакции 17 сентября 2018 г.)

\begin{abstract}
Проведено теоретическое исследование однопроходных лазеров на свободных электронах (ЛСЭ) с умножением гармоник в рентгеновском диапазоне с целью получения мощного излучения на наименьшей длине ЛСЭ с возможно более низкой энергией пучка и частотой затравочного лазера. Предложен каскадный ЛСЭ с двухчастотным ондулятором для группировки электронов на длинах волн высших гармоник. С помощью апробированной феноменологической модели ЛСЭ, которая учитывает все основные потери для каждой гармоники в каждом каскаде ЛСЭ, исследована динамика мощности излучения в ЛСЭ с каскадными умножителями гармоник. Изучено влияние качества пучка на генерацию рентгеновского излучения в ЛСЭ. Показано, что для эффективного использования гармоник в каскадном рентгеновском ЛСЭ нужны пучки электронов с разбросом энергии $\sigma_{e} \leq 0.0002$. Исследована эволюция мощности гармоник в нескольких каскадных ЛСЭ на длинах волн $\sim 2-3 \mathrm{~nm}$ с умножением гармоник затравочного лазера на длине волны $13.51 \mathrm{~nm}$ пика отражения $\mathrm{Mo} / \mathrm{Si}$ и на $11.43 \mathrm{~nm}$ пика отражения MoRu/Be; мощность достигла $\sim 1 \mathrm{GW}$ на длине ЛСЭ до $40 \mathrm{~m}$. Кроме того, исследована работа многокаскадных ЛСЭ с готовым затравочным эксимерным $\mathrm{UV}$ лазером на длине волны $157 \mathrm{~nm}$ ( $F_{2}$ excimer UV laser) и гармониками лазеров с $\mathrm{CO}_{2}$ и $\mathrm{C}_{2} \mathrm{H}_{2}$ на $30 \mathrm{~nm} \mathrm{c}$ пучком электронов энергии $\sim 0.6 \mathrm{GeV}$ и током $\sim 1 \mathrm{kA}$. Моделируемое в этих ЛСЭ рентгеновское излучение на длине волны $\lambda=2.5 \mathrm{~nm}$ и $\lambda=3.3 \mathrm{~nm}$ достигло мощности $\sim 0.5 \mathrm{GW}$ на $\sim 30 \mathrm{~m}$.
\end{abstract}

DOI: 10.21883/JTF.2019.03.47180.2600

\section{Введение}

Ондуляторное излучение (ОИ) основано на эффекте направленного в узкий угол $\sim 1 / \gamma$ интенсивного излучения ускоренных релятивистских зарядов энергии $E \gg m c^{2}$, где $\gamma=E / m c^{2}$ релятивистский параметр, $m$ - масса электрона и $c-$ скорость света. Его природа близка к синхротронному излучению (СИ), но ОИ происходит в пространственно-периодическом поле а не в однородном магнитном поле $[1,2]$. Спонтанное ОИ $[3,4]$ некогерентное, как и СИ. Спектр последнего квазинепрерывный и покрывает большую часть электромагнитного диапазона от радио до рентгеновских частот, а спектр ОИ дискретный и состоит из одного или нескольких резонансов. Источники когерентного излучения - лазеры - обычно работают на длинах волн более $200 \mathrm{~nm}$. Использование когерентного излучения уже на таких длинах волн позволяет исследовать вирусы, на длинах волн $\lambda \sim 14 \mathrm{~nm}$ различать квантовые загоны, а на длинах волн $\lambda \sim 1-2 \mathrm{~nm}$ изучать ДНК, углеродные нанотрубки и нановолокна. Для генерации столь коротковолнового когерентного излучения трудно найти подходящие для оптического резонатора материалы с хорошим коэффициентом отражения. В качестве источников излучения используют лазеры на свободных электронах (ЛСЭ) (см, например, [5-10]). Сама идея ЛСЭ была сформулирована Гинзбургом, который также предложил динамический ондулятор с движением электронного пучка в переменном во времени поле электромагнитной волны. Особенности получения мощного рентгеновского излучения освещены в [11-19]; для этого обычно применяют конструкцию ЛСЭ без зеркал с самоусилением спонтанного излучения (ССИ). Отсутствие зеркал снимает естественное ограничение ЛСЭ по мощности и частоте, но имеет и свои недостатки, в частности, отсутствие оптического резонатора не позволяет использовать его моды для задания структуры излучения. Кроме того, в ЛСЭ ССИ сам процесс генерации начинается с начального шума со случайной фазой, который приводит к постепенному образованию сгустков электронов, разделенных длиной волны излучения. Далее ОИ экспоненциально увеличивает свою интенсивность и становится все более и более когерентным, пока не наступит его насыщение. В режиме насыщения когерентность начинает разрушаться и происходит циклическая перекачка энергии от электромагнитной волны к электронам и наоборот. В однопроходных ЛСЭ весь вышеописанный процесс происходит за один проход излучения в длинном ондуляторе, на выходе которого можно получить серии сверхкоротких импульсов с пиковой мощностью до $\sim 10^{10} \mathrm{~W}$ с хорошей пространственной когерентностью. Рентгеновские ЛСЭ значительно превосходят мощность СИ в рентгеновском диапазоне $[8,13-19]$ и позволяют изучать физические, химические и биологические процессы на масштабе нанометра (см., например, [19]). Теория однопроходных ЛСЭ, которые также иногда называют параметрическими [20-22], подробно и доступно описана, например, в [12-15]. Самоусиление спонтанного излучения зарож- 
дается из случайного шума, что обусловливает слабую временную когерентность излучения на выходе ЛСЭ ССИ, т. е. время когерентности оказывается значительно меньше, чем полное время импульса ЛСЭ. Для преодоления этого недостатка можно использовать затравочное лазерное излучение на частоте ЛСЭ. В современных ЛСЭ, работающих в рентгеновском диапазоне, требуются пучки электронов высоких энергий, а сами ЛСЭ имеют огромные размеры. Так, например, недавно введенный в строй Европейский рентгеновский ЛСЭ (European XFEL) [23], используя основную гармонику, позволяет генерировать излучение длиной волны $<10^{-10} \mathrm{~m} \mathrm{и}$ имеет размер $3.4 \mathrm{~km}$. Это, в свою очередь, обусловливает очень высокую стоимость и сложность конструкции, в которой нужно поддерживать фокусировку пучка и взаимное пространственное положение электронов и фотонов на километровой длине.

Альтернативное решение, предложенное в [24,25], заключается в использовании высших гармоник ОИ в ЛСЭ с усилением высших гармоник (УВГ или $\mathrm{HGHG}$ ), сочетающее их генерацию, умножение, усиление и излучение. Для генерации гармоник в линейном режиме и группировки электронов на их длинах волн лучше всего подходят двухчастотные ондуляторы с двоякопериодическим магнитным полем [26-32]. В настоящей работе мы покажем несколько примеров использования таких ондуляторов в сочетании с высококачественными пучками электронов с $\gamma \sim 10^{3}$ и током $\sim 10^{2}$ А для генерации десятков мегаватт нанометрового излучения в ЛСЭ с ондуляторами длиной всего $35-40 \mathrm{~m}$. Это короче или сопоставимо с длиной устройств, исследованных в [33-35], но при этом предлагаемый нами ЛСЭ имеет меньшую длину волны, $\lambda \approx 2-4 \mathrm{~nm}$. Кроме того, каскадные ЛСЭ с умножением гармоник имеют важное преимущество: они позволяют использовать электроны меньших энергий и затравочный лазер более низкой частоты, чем частота излучения ЛСЭ. Мы покажем это на примере ЛСЭ с готовым затравочным $F_{2}$ эксимерным UV-лазером. Использование высококачественного затравочного источника позволяет получить излучение ЛСЭ со стабильными характеристиками и отличной временной когерентностью в дополнение к высокой мощности ЛСЭ ССИ.

Ниже мы исследуем динамику мощности ЛСЭ с помощью разработанной нами ранее аналитической модели.

\section{Аналитическая модель однопроходного каскадного ЛСЭ}

Теория взаимодействия излучения с электронами в ЛСЭ была развита во второй половине прошлого века Мадэем [36]. Подробный обзор и доступное описание работы однопроходного ЛСЭ содержится, например, в работах [11-15]. На основе аналитического описания физических процессов в ЛСЭ в [37-39] была развита модель однопроходного каскадного ЛСЭ с учетом линейной и нелинейной генерации гармоник. Корректное моделирование поведения высших гармоник необходимо для каскадных ЛСЭ и ЛСЭ с УВГ, поэтому феноменологическая модель была дополнена учетом основных потерь для каждой гармоники в каждом каскаде в [40]. Высшие гармоники более чувствительны к потерям и не идеальностям пучка и ондулятора, чем основная гармоника. В квазианалитическом описании нами использовались основные зависимости поведения коэффициентов потерь, взятые из предыдущих исследований и точных расчетов с обобщенными функциями типа Эйри и Бесселя [26,27,31,42,43]. Мы предполагаем согласованный с ондулятором пучок; исследование вопросов фокусировки находится за рамками нашей модели и может быть проделано с помощью формул из [44-48] или соответствующих компьютерных программ.

Мощность гармоник излучения в ЛСЭ с изначально не сгруппированным пучком электронов растет экспоненциально по длине ондуляторов [11-15] от 1/9 затравочной мощности $P_{0, n}$ до мощности насыщения $P_{n . F}=\frac{P_{F}}{\sqrt{n}}\left(\frac{f_{n}}{n f_{1}}\right)^{2}$. Этот рост мощности может быть аппроксимирован следующим образом [30]:

$$
\begin{aligned}
P_{L, n}(z) \cong & \frac{P_{0, n} A(n, z) \exp \left(0.223 z / Z_{s}\right)}{1+(A(n, z)-1) P_{0, n} / P_{n, F}}, \\
A(n, z) \cong \frac{1}{3} & +\frac{1}{4.5} \cosh \frac{z}{L_{n, g}} \\
& +\frac{5}{2.22} \cos \frac{\sqrt{3} z}{2 L_{n, g}} \cosh \frac{z}{2 L_{n, g}},
\end{aligned}
$$

где $L_{n, g} \cong \lambda_{u} /\left(4 \pi \sqrt{3} n^{1 / 3} \rho_{n}\right)$ - длина усиления, $Z_{s} \cong 1.07 L_{1, g} \ln \left(9 P_{F} / P_{0,1}\right)$ - длина насыщения, $P_{F} \cong$ $\sqrt{2} \rho_{1} P_{e}, \quad \lambda_{u}-$ основной период ондулятора [M], $P_{e}-$ мощность электронного пучка, $\rho_{n}=\frac{1}{2 \gamma} \times$ $\times\left(\frac{J}{2 \pi i}\left(\lambda_{u} \tilde{k} f_{n}\right)^{2}\right)^{1 / 3}$ - параметр Пирса (см., например, $[14]), \tilde{K}=k / \sqrt{2}$ - эффективный ондуляторный параметр [31], $f_{n}-$ коэффициенты Бесселя для гармоники $n$ ОИ (см., например, [30,40]), $J$ - плотность электронного тока $\left[\mathrm{A} / \mathrm{m}^{2}\right], i \cong 1.7 \cdot 10^{4}$ - значение тока Альфвена $[\mathrm{A}]$. Для ондулятора ЛСЭ, получающего уже сгруппированный пучок, рост мощности излучения начинается без задержки [45]:

$$
\begin{gathered}
P_{L, n}(z) \cong \frac{P_{0, n} F(n, z)}{1+F(n, z) P_{0, n} / P_{F, n}}, \\
F(n, z) \cong 2\left|\cosh \left(z / L_{n, g}\right)-\cos \left(z / 2 L_{n, g}\right) \cosh \left(z / 2 L_{n, g}\right)\right|,
\end{gathered}
$$

где $P_{0, n}-$ начальная мощность гармоники номера $n$, которая приходит из предыдущей секции, или эквивалентная мощность за счет банчинга с учетом его ренормализации множителем $\left(\rho_{1, \mathrm{sec} 1} / \rho_{1, \mathrm{sec} 2}\right)^{k}$ для отличающихся друг от друга ондуляторов в соседних секциях, и $k-$ отношение гармоник в них. Линейную генерацию 
гармоник (1), (2) дополняет нелинейная, при которой мощность гармоник растет как $n$-я степень мощность основного тона [30]:

$$
Q_{n}(z) \cong P_{n, 0} \frac{\exp \left(n z / L_{g}\right)}{1+\left(\exp \left(n z / L_{g}\right)-1\right) P_{n, 0} / P_{n, F}},
$$

где $L_{g} \cong L_{1, g}, P_{n, 0} \cong d_{n} b_{n}^{2} P_{n, F}-$ эквивалентная затравочная мощность за счет банчинга, $d_{3} \cong 8, d_{5} \cong 116$, $b_{n}$ - индуцированные основной гармоникой ЛСЭ коэффициенты группировки, которые эволюционируют по длине ЛСЭ следующим образом: $b_{n}(z)=$ $=h_{n}\left(P_{1}(z) / P_{e} \rho_{1}\right)^{n / 2}, h_{1,2,3,4,5}=\{1,1.5,2.5,4.5,7.5\}[38]$. Полная мощность излучения гармоник складывается из их линейной и нелинейной составляющих: $P_{n}=P_{L, n}+Q$. Коэффициенты Бесселя $f_{n}[30]$ для обычного плоского ондулятора (см. (4) с $d=0$ ), $f_{n, z}=J_{\frac{n-1}{2}}(\xi)-J_{\frac{n+1}{2}}(\xi)$ содержат обычные функции Бесселя $J_{n}(\xi)$, где $\xi=n k^{2} / 4\left(1+\frac{k^{2}}{2}\right)$.

Плоский двухчастотный ондулятор [26,27] с магнитным полем

$$
\begin{gathered}
\widehat{H}=\left(0, H_{0}\left(\sin \left(k_{\lambda} z\right)+d \sin \left(h k_{\lambda} z\right)\right), 0\right), \\
k_{\lambda}=2 \pi / \lambda_{u}, \quad h \in Z, \quad d, h=\mathrm{const}
\end{gathered}
$$

позволяет получить значительное усиление и большие коэффициенты группировки для высших гармоник ЛСЭ [40,49]; его коэффициенты Бесселя $f_{n, x}=I_{n-1}^{(h)}(n)+I_{n+1}^{(h)}(n)+\frac{d}{h}\left(I_{n+h}^{(h)}(n)+I_{n-h}^{(h)}(n)\right)$ выражаются через обобщенные функции Бесселя (см. $[27,28,42,43])$, которые имеют следующий вид:

$$
\begin{aligned}
& I_{n}^{(h)}(m)=\int_{0}^{2 \pi} \cos [n \phi+m \\
& \left.\times \frac{k^{2}\left(\frac{\sin 2 \phi}{4}+\frac{d \sin (h-1) \phi}{h(h-1)}+\frac{d \sin (h+1) \phi}{h(n+1)}+\frac{d^{2} \sin 2 h \phi}{4 h^{3}}\right)}{1+\left(k^{2}+k_{2}^{2}\right) / 2}\right] \frac{d \phi}{2 \pi},
\end{aligned}
$$

где $k_{2}=k|d / h|$. Для обычного ондулятора нужно считать $d=0$ во всех приведенных выше выражениях. Эллиптические двухчастотные ондуляторы в отличие от плоских ондуляторов позволяют эффективно регулировать поляризацию, но предоставляют меньше возможностей по управлению интенсивностью гармоник [30].

Для описания дополнительного вклада от роста начального шума электронного банча, вдобавок к основному росту мощности затравочного лазерного излучения, подходит следующая приближенная формула:

$$
\begin{gathered}
N_{L, n}(z) \approx \frac{P_{\text {noise }} S(n, z) / 9}{1+S(n, z) / 45}, \\
S(n, z)=2\left|\left(\cosh \frac{z}{L_{n, g}}-\cos \frac{z}{20 L_{n, g}} \cosh \frac{z}{2 L_{n, g}}\right)\right|,
\end{gathered}
$$

где $P_{\text {noise }}$ - мощность шума банча, функция $N_{L, n}(z)$ выбрана с учетом численных симуляций в [41] и влияет на мощность в начале ЛСЭ.
Приведенная выше модель описывает работу ЛСЭ в идеальном случае. В реальности дифракция пучка несколько уменьшает значение параметра Пирса по следующей формуле [38]:

$$
\rho_{n} \rightarrow \rho_{D, n}=\frac{\rho_{n}}{\left(1+\mu_{D, n}\right)^{1 / 3}}, \quad \mu_{D, n}=\frac{\lambda_{u} \lambda_{n}}{(4 \pi)^{2} \rho_{n} \sigma},
$$

где $\lambda_{n}=\frac{\lambda_{u}}{2 n \gamma^{2}}\left(1+\frac{k_{e f f}^{2}}{2}\right)$ - длина волны гармоники излучения, $\Sigma=\pi \sigma=\frac{P_{e}}{E_{e} \gamma J}-$ сечение гауссова электронного пучка, $E_{e}=m_{e} c^{2} \cong 0.51 \cdot 10^{6} \mathrm{eV}-$ энергия покоя электрона, $P_{e}-$ мощность пучка, $J=I_{0} /(\pi \sigma)$ - плотность тока в гауссовом пучке, $I_{0}$ - электронный ток. Разброс энергии и расходимость пучка увеличивают длину насыщения $L_{n, g} \rightarrow L_{n, g} \Phi$ и уменьшают мощность насыщения $P_{n, F} \rightarrow n P_{n, F} \eta$; коррекция может достигать $30 \%$. При этом мы феноменологически учитываем номер гармоники $\mathrm{n}$ в корректирующих коэффициентах для каждого ондуляторного каскада:

$$
\begin{gathered}
\mu_{\varepsilon, n}\left(\sigma_{\varepsilon}, n\right) \cong 2 n \sigma_{\varepsilon} /\left(n^{1 / 3} \rho_{n}\right), \\
\xi \cong 1+0.07 \sum_{i} \mu_{i}+0.35 \sum_{i} \mu_{i}^{2},
\end{gathered}
$$

где $\mu_{i}=\mu_{x, y}$ (см. [39]), и в выражениях $\Phi_{n}\left(\sigma_{e}, \zeta, n\right)$ и $\eta_{n}\left(\sigma_{\varepsilon}, \zeta, n\right)$ :

$$
\begin{gathered}
\Phi_{n} \cong\left(\zeta^{n^{2 / 3}}+0.165 \mu_{\varepsilon, n}^{2}\right) e^{0.034 \mu_{\varepsilon, n}^{2}}, \\
\eta_{n} \cong\left(e^{-\Phi_{n}\left(\Phi_{n}-0.9\right)}+1.57\left(\Phi_{n}-0.9\right) / \Phi_{n}^{3}\right) / 1.062 .
\end{gathered}
$$

Зависимость $\zeta$ от параметров Твисса достаточно громоздкая; она приведена в $[39,45,49]$ и не меняет существа формул (8),(9); с хорошим пучком в рентгеновском диапазоне $\xi \leq 1.04$.

Важнейшим параметром источника излучения является его спектральная яркость, т.е. число фотонов в единицу времени в спектральном диапазоне $\Delta \lambda / \lambda$ на единицу площади $S$ в единицу телесного угла $\Omega$. При оценке яркости источника ОИ нужно учитывать его эффективный поперечный размер $\Delta_{x, y}=\sqrt{\left(\lambda_{u} L_{u}\right) /(4 \pi)^{2}+\sigma_{x, y}^{2}+\theta_{x, y}^{2} L_{u}^{2}}$ и эффективную расходимость излучения в угол $\delta_{x, y}=\sqrt{\lambda / L_{u}+\theta_{x, y}^{2}}$, где $L_{u}$ - длина ондулятора, $\sigma_{x, y}-$ поперечный размер электронного пучка, $\theta_{x, y}$ - угловая расходимость пучка, $\lambda$ - длина волны излучения. Эти параметры связаны между собой через эмиттанс $\varepsilon_{x, y}=\sigma_{x, y} \theta_{x, y}$ и параметры Твисса $\beta_{x, y}: \sigma_{x, y}=\sqrt{\varepsilon_{x, y} \beta_{x, y}}$ и $\theta_{x, y}=\sqrt{\varepsilon_{x, y} / \beta_{x, y}}$. Сечение гауссова пучка $\Sigma=\pi r^{2}$, где $r-$ его средний радиус, определяется следующим образом: $\Sigma=2 \pi \sqrt{\beta_{x} \varepsilon_{x}^{n} \beta_{y} \varepsilon_{y}^{n}} / \gamma$, где $\varepsilon_{x, y}^{n}=\gamma \varepsilon_{x, y}$ - нормализованный эмиттанс. Используя пучки высоких энергий с малым эмиттансом, можно применять длинные ондуляторы для генерации коротковолнового излучения. Для реализации пространственнокогерентного источника излучения необходимо, чтобы 
его фазовый объем был меньше дифракционного предела: $\Delta_{x, y} \delta_{x, y} \leq \lambda / 4 \pi$, что дает условие для стабильной работы ЛСЭ, $\varepsilon_{x, y} \leq \lambda_{0} / 4 \pi$. Например, для генерации на длине волны $1 \mathrm{~nm}$ имеем $\varepsilon_{x, y}<0.1 \mathrm{~nm} \cdot \mathrm{rad}$. Другим условием генерации в ЛСЭ является малый разброс энергий $[14,15] \sigma_{e} \leq \rho / 2$. Современные ускорителирекуператоры электронов позволяют получить высоко моноэнергетические пучки с $\varepsilon_{x, y}<0.01 \mathrm{~nm} \cdot \mathrm{rad}$ и $\sigma_{e} \approx 10^{-4}$. В результате взаимодействия излучения с банчем увеличивается разброс энергий электронов за счет индуцированного основным тоном ЛСЭ разброса вдобавок к начальному разбросу $\sigma_{e, 0}[45]$ :

$$
\begin{aligned}
& \sigma_{e}^{2}(z) \cong \sigma_{e, 0}^{2} \\
& +\frac{3}{2} \sqrt{\frac{\left.A(1, z)\right|_{\rho \rightarrow \rho_{D, 1}} \rho_{D, 1} P_{0} / P_{e}}{1+1.24\left(\left.A(1, z)\right|_{\rho \rightarrow \rho_{D, 1}}-1\right) P_{0} / P_{1, F} \eta\left(\mu_{\varepsilon, 1}\right)}} .
\end{aligned}
$$

Приведенная выше аналитическая модель ЛСЭ хорошо воспроизводит эволюцию мощности не только основной, но и высших гармоник ЛСЭ, что важно для моделирования ЛСЭ с УВГ и каскадного ЛСЭ с умножением гармоник, которое мы проведем в следующих разделах.

\section{ЛСЭ с умножением высших гармоник в нанометровом диапазоне}

Исследуем генерацию нанометрового излучения от электронов с энергией $E \sim 1 \mathrm{GeV}$ в каскадном ЛСЭ. Мощность излучения гармоник ЛСЭ с двухчастотным ондулятором на длине волны $1-100 \mathrm{~nm}$ может достигать $\sim 100 \mathrm{~kW}[40]$, что значительно превышает мощность гармоник обычных лазеров в этом диапазоне длин волн. Важным преимуществом двухчастотного ондулятора по сравнения с обычным является то, что он интенсивно излучает гармоники и производит группировку электронов на их длинах волн, $\lambda_{n}=\lambda_{0} / n$. В обычном ондуляторе группировка в основном происходит на длине основной гармоники излучения ЛСЭ, $\lambda_{0}$. По сравнению с обычным ондулятором, где $d=0$ в (4), двухчастотный ондулятор может давать до 10 раз большее значение банчинга для гармоник в линейном режиме при правильном выборе значений $d$ и $h$ в (4). Поэтому двухчастотный ондулятор может работать как эффективный группирователь электронов непосредственно на длинах волн гармоник, что важно в ЛСЭ с УВГ (HGHG FEL). Чтобы усилить высшие гармоники двухчастотного ондулятора, выберем $h=3, d=0.5$ следуя $[31,42,43]$ для ондулятора c $k=2.133, \lambda_{u 1}=2.8 \mathrm{~cm}$ в группирователе электронов. В каскаде усилителя-излучателя, настроенного на пятую гармонику двухчастотного группирователя, выберем $k_{2}=1, \lambda_{u 2}=1.25 \mathrm{~cm}$. Более слабые ондуляторы с большим периодом увеличат длину ЛСЭ. Длина волны затравочного излучения $13.51 \mathrm{~nm}$ соответствует пику отражения покрытия из $\mathrm{Mo} / \mathrm{Si}[50]$. С учетом индуцированного ЛСЭ разброса энергий (10) точку разрыва каскадов выберем из условия $\sigma_{\varepsilon} \leq \rho_{\mathrm{sec} 2} / 2$. Моделирование выполнено в программе Mathematica. Рост мощности гармоник в ЛСЭ с УВГ с электронным пучком мощности $E=950 \mathrm{MeV}$ и разбросом энергий $\sigma_{e}=0.0001$ показан на рис. 1. Некоторые данные моделирования приведены ниже.

$f_{n, 1}=\{0.756,0.339,0.201\}, f_{n, 2}=\{0.901,0.212,0.070\}$, $\rho_{D, n, 1}=\{0.0011,0.0007,0.0005\}$,

$\rho_{D, n, 2}=\{0.00048,0.00019,0.00009\}$,

$E=950 \mathrm{MeV}, \quad \gamma=1860, \quad P_{E}=1000 \mathrm{GW}$,

$J=1 \times 10^{10} \mathrm{~A} / \mathrm{m}^{2}, \quad \Sigma=1 \times 10^{-7} \mathrm{~m}^{2}, \quad I_{0}=1.05 \mathrm{kA}$,

$\xi=1.05, \quad \sigma_{e}=0.0001, \quad k=2.133, \quad h=3, \quad d=0.5$,

$\lambda_{u, 1}=2.8 \mathrm{~cm}, L_{s}=22 \mathrm{~m}$,

$L_{\text {gain } 1}=1.1 \mathrm{~m}, k_{2}=1, \lambda_{u, 2}=1.25 \mathrm{~cm}, L_{s 2}=20 \mathrm{~m}$,

$L_{\text {gain } 2}=1.5 \mathrm{~m}, \lambda_{\mathrm{sec} 1}=13.51 \mathrm{~nm}, \lambda_{\mathrm{sec} 2}=2.71 \mathrm{~nm}$,

$\lambda_{\mathrm{sec} 3, n=3}=0.9 \mathrm{~nm}, \lambda_{\mathrm{sec} 1} / \lambda_{\mathrm{sec} 2}=5$.

Здесь и далее мы закладываем 20\% потерь эффективной мощности между каскадами; расстояния между ондуляторами следует добавить к полной длине ондуляторов ЛСЭ, как и в последующих примерах. Мощность насыщения на длине волны излучения основного тона $\lambda_{\mathrm{sec} 2}=2.71 \mathrm{~nm}$ усилителя ЛСЭ (длинная пунктирная линия) на длине $\approx 45 \mathrm{~m}$ составляет $\approx 600 \mathrm{MW}$ (рис. 1). Третья гармоника излучателя на длине волны $\lambda_{3}=0.9 \mathrm{~nm}$ (пунктирная линия) полностью индуцирована первой гармоникой; ее мощность достигает $\approx 350 \mathrm{~kW}$. Отметим, что даже очень малый разброс энергий электрона, $\sigma_{e}=0.0001$, влияет на работу ЛСЭ: при меньшем значении, например $\sigma_{e}=0.00005$, длина ЛСЭ сократилась бы на несколько метров, а мощность пятой гармоники группирователя в точке разрыва была бы выше. Соответствующий график мы не приводим для краткости также потому, что значение $\sigma_{e}=0.00005$ в настоящее время практически еще не достижимо. Вклад начального шума электронного банча влияет на мощность на первых метрах и несущественен в точке разрыва группирователя; здесь и в дальнейшем мы не представляем его на графиках.

Мы сравнили работу ЛСЭ с обычным ондулятором в группирователе электронов с $k=2.133$, $\lambda_{u, 1}=2.8 \mathrm{~cm}$ (сплошные линии на рис. 2) и с двухчастотным ондулятором-группирователем с $k=2.133$, $\lambda_{u, 1}=2.8 \mathrm{~cm}, d=3, h=0.5$ (см. (4)) (пунктирные линии на рис. 2). Уже при разбросе энергий $\sigma_{e}=0.0002$ обычный ондулятор очень слабо усиливает высшие гармоники, так что мощность пятой гармоники в нем (нижняя пунктирная линия на рис. 2) почти не растет, и на выходе группирователя составляет менее $10^{-2} \mathrm{~W}$. Некоторые данные моделирования мощности гармоник в ЛСЭ с УВГ при $\sigma_{e} \approx 0.0002$ (рис. 2) с затравочной длиной волны $\lambda_{\mathrm{sec} 1}=11.43 \mathrm{~nm}$ приведены ниже.

$f_{n, 1}=\{0.756,0.339,0.201\}$, $f_{n, 2}=\{0.910,0.212,0.070\}$ $\rho_{n, 1}=\{0.0009,0.0006,0.0004\}$ $\rho_{n, 2}=\{0.0004,0.0002,0.00007\}$, 

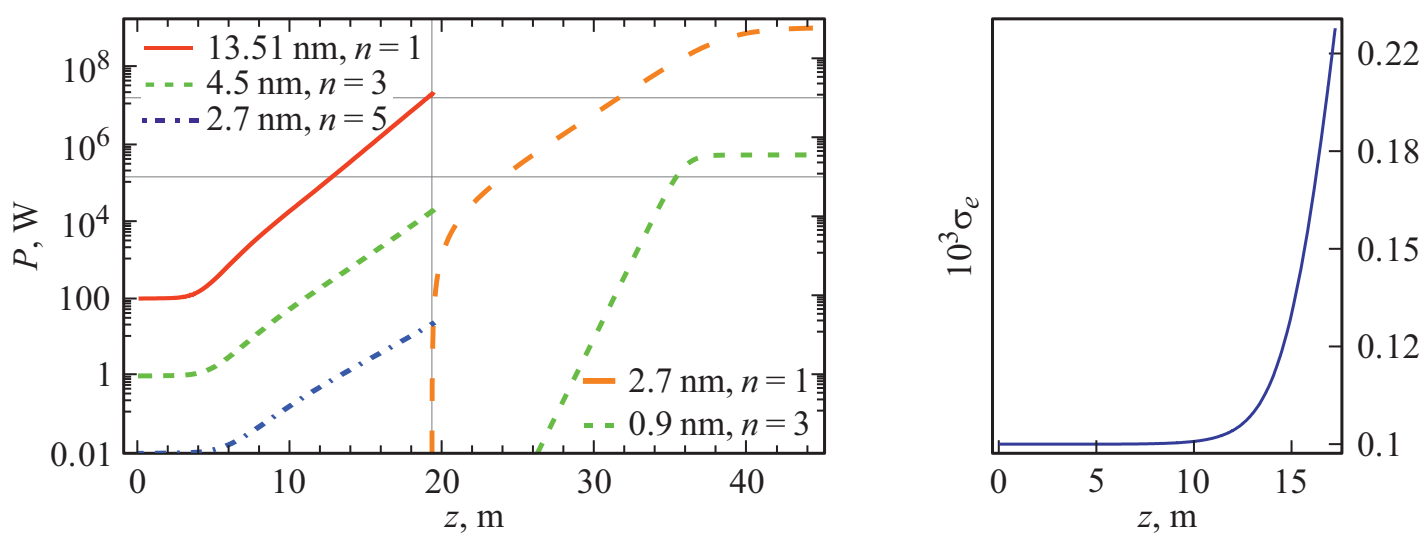

Рис. 1. Эволюция мощности гармоник в ЛСЭ с УВГ (HGHG FEL) с двухчастотным группирователем электронов энергии $E=950 \mathrm{MeV}$ и разбросом энергий $\sigma_{e}=0.0001$ (левый график); эволюция разброса энергий по длине группирователя электронов (правый график).

$P_{E}=92.4 \mathrm{GW}, J=9.96 \cdot 10^{9} \mathrm{~A} / \mathrm{m}^{2}$,

$\sigma=2.86 \cdot 10^{-9} \mathrm{~m}^{2}, I_{0}=89 \mathrm{~A}, \sigma_{e}=0.00017, \xi=1.03$,

$k=2.133, h=3, d=-0.5, \lambda_{u, 1}=2.8 \mathrm{~cm}, L_{s}=31.4 \mathrm{~m}$,

$L_{\text {gain }}=1.42 \mathrm{~m}, k_{2}=1, \lambda_{u, 2}=1.25 \mathrm{~cm}, L_{s, 2}=24.5 \mathrm{~m}$,

$L_{\mathrm{gain}, 2}=1.73 \mathrm{~m}, \lambda_{\mathrm{sec} 1}=11.43 \mathrm{~nm}, \lambda_{\mathrm{sec} 2}=2.29 \mathrm{~nm}$,

$\lambda_{\mathrm{sec} 1} / \lambda_{\mathrm{sec} 2}=5, \lambda_{n=3, \sec 2}=0.76 \mathrm{~nm}$.

Мощность пятой гармоники двухчастотного группирователя в точке разрыва сопоставима с мощностью третьей гармоники обычного ондулятора (ср. среднюю пунктирную и нижнюю сплошную линии в левой части рис. 2). Высшие гармоники в двухчастотном ондуляторе при $\sigma_{e}=0.0002$, хотя и более слабые, чем при $\sigma_{e}=0.0001$, могут использоваться в ЛСЭ с УВГ, как показано на рис. 2. Отметим также несколько меньшее усиление основного тона в группирователе с двухчастотном ондуляторе по сравнению с обычным ондулятором (ср. сплошную и пунктирную верхние линии в левой части рис. 2. В ЛСЭ с УВГ это является преимуществом, так как сопутствующий рост разброса энергий пучка по длине ЛСЭ индуцируется наиболее мощным основным тоном. Таким образом, с использованием гармоник двухчастотного группирователя индуцированный разброс энергий оказывается меньшим, чем с использованием обычного плоского ондулятора в группирователе.

Чем слабее гармоники группирователя, тем более длинным должен быть усилитель-излучатель; так, при $\sigma_{e}=0.0002$ полная длина ондуляторов ЛСЭ достигает $\sim 55 \mathrm{~m}$ (рис. 2), а при $\sigma_{e}=0.0001$ достаточно $\approx 45 \mathrm{~m}$. При большем разбросе энергий пучка сильно уменьшается выходная мощность гармоник усилителя ЛСЭ: при $\sigma_{e}=0.00017$ третья гармоника ЛСЭ достигает $\sim 23 \mathrm{~kW}$ (рис. 2 ) против $\approx 100 \mathrm{~kW}$ для $\sigma_{e}=0.0001$. Большая длина короткопериодного излучателя делает более сложным и дорогим его изготовление. Кроме того, поддержание фокусировки пучка и пространственное согласование банча электронов и импульса фотонов в длинном излучателе представляет дополнительную проблему.

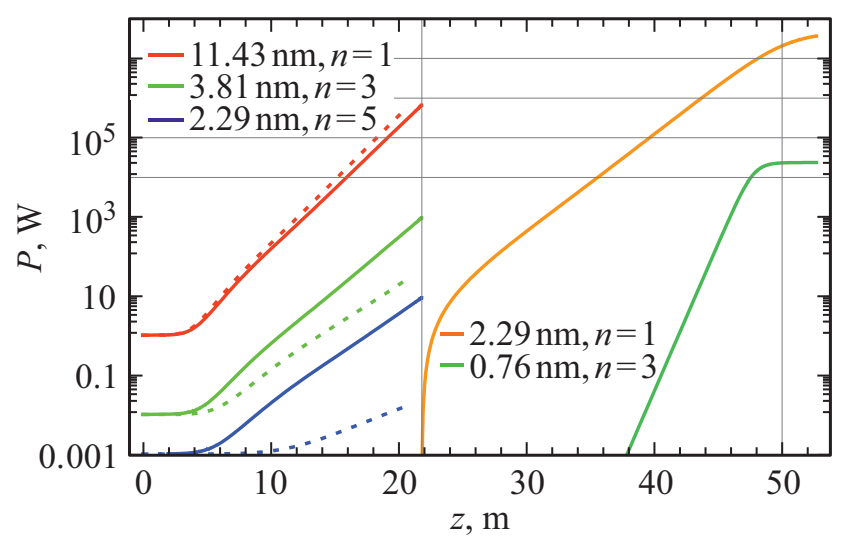

Рис. 2. Эволюция мощности гармоник в ЛСЭ с УВГ (HGHG FEL) с пучком электронов энергии $E=950 \mathrm{MeV}$ и разбросом энергий $\sigma_{e}=0.00017$. В группирователе электронов сплошные линии - двухчастотный плоский ондулятор с $d=3, h=0.5$, пунктирные линии - обычный плоский ондулятор $(d=0$ в (4)).

Отметим, что выше мы использовали длины волн затравочного излучения 11.43 и $13.51 \mathrm{~nm}$, соответствующие пикам отражения $\mathrm{MoRu} / \mathrm{Be}$ и $\mathrm{Mo} / \mathrm{Si}$; детальное исследование и выбор затравочных лазеров остается за рамками нашего исследования. Использование мод оптических резонаторов позволяет стабилизировать фазу и получить заданные пространственно-временные характеристики излучения ЛСЭ с УВГ в отличие от ЛСЭ с ССИ, где генерация начинается с шума со случайной фазой. Излучение ЛСЭ с ССИ имеет хорошую пространственную структуру и большую мощность, но слабую временную когерентность. В отсутствие готовых затравочных лазеров на высокой частоте можно использовать самозатравочные ЛСЭ [51,52] или высшие гармоники, сгенерированные в лазерах с молекулами $\mathrm{F}_{2}$, $\mathrm{CO}_{2}, \mathrm{C}_{2} \mathrm{H}_{2}[53]$. 


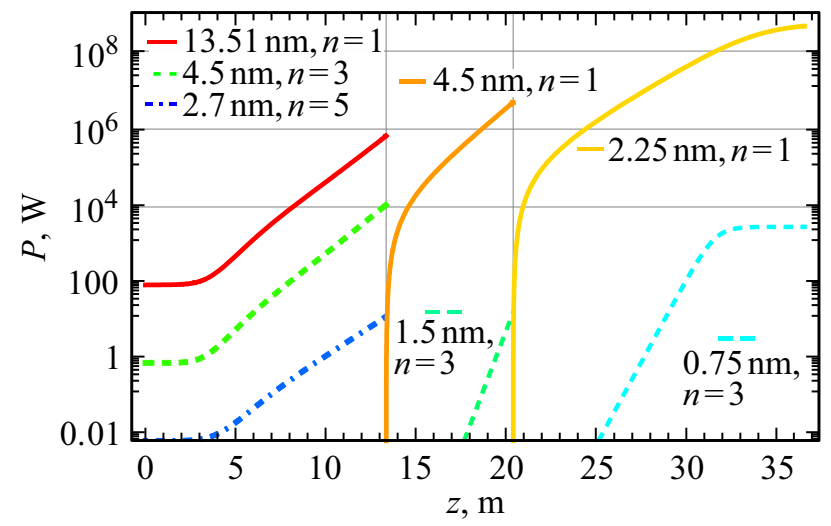

Рис. 3. Эволюция мощности гармоник в трехкаскадном ЛСЭ с затравочным лазером на $13.51 \mathrm{~nm}$, двухчастотным группирователем, умножителем третьей гармоники группирователя и усилителем второй гармоники умножителя; начальный разброс энергий пучка: $\sigma_{e}=0.0002$.

\section{Многокаскадные ЛСЭ для мягкого рентгеновского излучения}

Выше мы показали, что для получения нанометрового излучения в каскадном ЛСЭ необходимы пучки электронов очень высокого качества; уже при разбросе энергий электронов $\sigma_{e}=0.0002$ использование высших гармоник двухчастотных ондуляторов становится затруднительным, а гармоник обычных ондуляторов в группирователях - практически неэффективным (рис. 2). Кроме того, длина излучателя ЛСЭ при $\sigma_{e}=0.0002$ увеличивается по сравнению с его длиной при $\sigma_{e}=0.0001$, что создает массу технических проблем и значительно увеличивает стоимость конструкции. При $\sigma_{e}>0.0001$ в каскадных ЛСЭ целесообразно использовать более низкие гармоники ондуляторов, которые менее подвержены негативному влиянию разброса энергий и расходимости пучка. Чтобы получить излучение ЛСЭ на $\lambda \approx 2.27 \mathrm{~nm} \mathrm{c}$ пучком с разбросом энергий $\sigma_{e}=0.0002$ можно ввести дополнительный каскад и использовать достаточно сильную третью гармонику двухчастотного группирователя. Эволюция мощности гармоник ЛСЭ представлена на рис. 3. Некоторые детали моделирования приведены ниже.

$f_{n, 1}=\{0.756,0.339,0.201\}, f_{n, 2}=\{0.845,0.298,0.153\}$, $f_{n, 3}=\{0.923,0.188,0.055\}$,

$\rho_{n, 1}=\{0.0012,0.0007,0.0005\}$,

$\rho_{n, 2}=\{0.0007,0.0003,0.0002\}$,

$\rho_{n, 3}=\{0.0004,0.00014,0.00006\}$,

$\gamma=1860, P_{e}=1000 \mathrm{GW}, J=1.0 \cdot 10^{10} \mathrm{~A} / \mathrm{m}^{2}$,

$\Sigma=1.05 \cdot 10^{-7} \mathrm{~m}^{2}, I_{0}=1.05 \mathrm{kA}, \sigma_{e}=0.0002, \xi=1.05$, $k=2.1330, h=3, d=0.5, \lambda_{u, 1}=2.8 \mathrm{~cm}, L_{s}=24 \mathrm{~m}$, $L_{\text {gain }}=1.2 \mathrm{~m}, \quad k_{2}=1.5655, \lambda_{u, 2}=1.4 \mathrm{~cm}, \quad L_{s, 2}=17 \mathrm{~m}$, $L_{\text {gain, } 2}=1.0 \mathrm{~m}, k_{3}=0.9, \lambda_{u, 3}=1.12 \mathrm{~cm}, L_{s, 3}=13 \mathrm{~m}$,

$L_{\text {gain }, 3}=1.5 \mathrm{~m}, \lambda_{\mathrm{sec} 1}=13.51 \mathrm{~nm}, \lambda_{\mathrm{sec} 2}=4.5 \mathrm{~nm}$,

$\lambda_{\sec 1} / \lambda_{\sec 2}=3, \lambda_{\sec 3}=2.25 \mathrm{~nm}, \lambda_{\sec 2} / \lambda_{\sec 3}=2$.
Используя трехкаскадный ЛСЭ с суммарным коэффициентом умножения гармоник на шесть и пучок электронов энергии $950 \mathrm{MeV}$ с разбросом $\sigma_{e}=0.0002$, получаем с затравочным излучением на длине волны $13.51 \mathrm{~nm}$ максимума отражения $\mathrm{Mo} / \mathrm{Si} \approx 500 \mathrm{MW}$ излучения на длине волны $\lambda_{\mathrm{sec} 3}=2.25 \mathrm{~nm}$ на $\sim 36 \mathrm{~m}$ длины ЛСЭ. Это короче, чем в двухкаскадном ЛСЭ на пятой гармонике группирователя с вдвое меньшим разбросом энергий $\sigma_{e}=0.0001$ и большей длиной волны $\lambda=2.7 \mathrm{~nm}$ (cp. рис. 1). Промежуточный каскад умножителя гармоник настроен на третью гармонику двухчастотного группирователя электронов на $\lambda_{\mathrm{sec} 2}=4.5 \mathrm{~nm}$. Усилительизлучатель настроен на вторую, не излучаемую гармонику умножителя на $\lambda_{\mathrm{sec} 2, n=2}=2.25 \mathrm{~nm}$, в котором основной тон (рис. 3 ) индуцирует банчинг на высших гармониках и этим дает эквивалентную затравочную мощность для последнего каскада излучателя. Таким образом, в трехкаскадном ЛСЭ с использованием гармоник $n=3$ и $n=2$ (рис. 3) достигается та же мощность на примерно той же частоте, что и в ЛСЭ с УВГ с $n=5$ на рис. 1, но с вдвое меньшим разбросом энергий. Подчеркнем, что такой ЛСЭ менее чувствителен к разбросу энергий пучка электронов из-за использования более низких гармоник; требуемый коэффициент умножения гармоник достигается с большим числом каскадов. Поскольку изза разброса энергий индуцированная третья гармоника умножителя - пунктирная линия во втором каскаде довольно слабая, то последний каскад настроен на вторую, не излучаемую в умножителе гармонику. Эквивалентная мощность на ее длине волны формируется исключительно за счет банчинга и ее величина где-то посередине между мощностью первой и третьей гармоник умножителя, т.е. между сплошной и пунктирной линиями второго каскада на рис. 3. Важно также то, что длина дорогого короткопериодного излучателя в трехкаскадном ЛСЭ с умножением гармоник существенно сокращается за счет лучшей группировки электронов в предыдущих каскадах, и составляет $<17 \mathrm{~m}$. Это даже короче, чем длина излучателя в ЛСЭ с УВГ с разбросом энергий $\sigma_{e}=0.0001$ (ср. с рис. 1). Для пучка с $\sigma_{e}=0.0002$, использованного в ЛСЭ на рис. 2, в ЛСЭ с УВГ потребовался бы излучатель длиной $\sim 30 \mathrm{~m}$. Точки разрыва каскадов выбраны на 13.4 и на $20.4 \mathrm{~m}$ на основе расчета индуцированного ЛСЭ разброса энергии в каждом каскаде, представленного на рис. 4.

Многокаскадный ЛСЭ с умножением гармоник обычных лазеров, например, с $\lambda_{\text {seed }}=30 \mathrm{~nm}$ и мощностью $1 \mathrm{~kW}$ [53], может быть использован для получения мощного когерентного рентгеновского излучения. С высококачественным пучком относительно низко энергичных электронов энергии $E=638 \mathrm{MeV}$ возможна генерация излучения на длине волны $\lambda_{\mathrm{sec} 4}=2.5 \mathrm{~nm}$ мощностью $\approx 400 \mathrm{MW}$ уже на $30 \mathrm{~m}$. В рассмотренном нами ЛСЭ предлагается двухчастотный ондулятор в группирователе электронов для их эффективной группировки на длине волны третьей гармоники в линейном режиме с минимальным индуцированным ЛСЭ разбросом энер- 

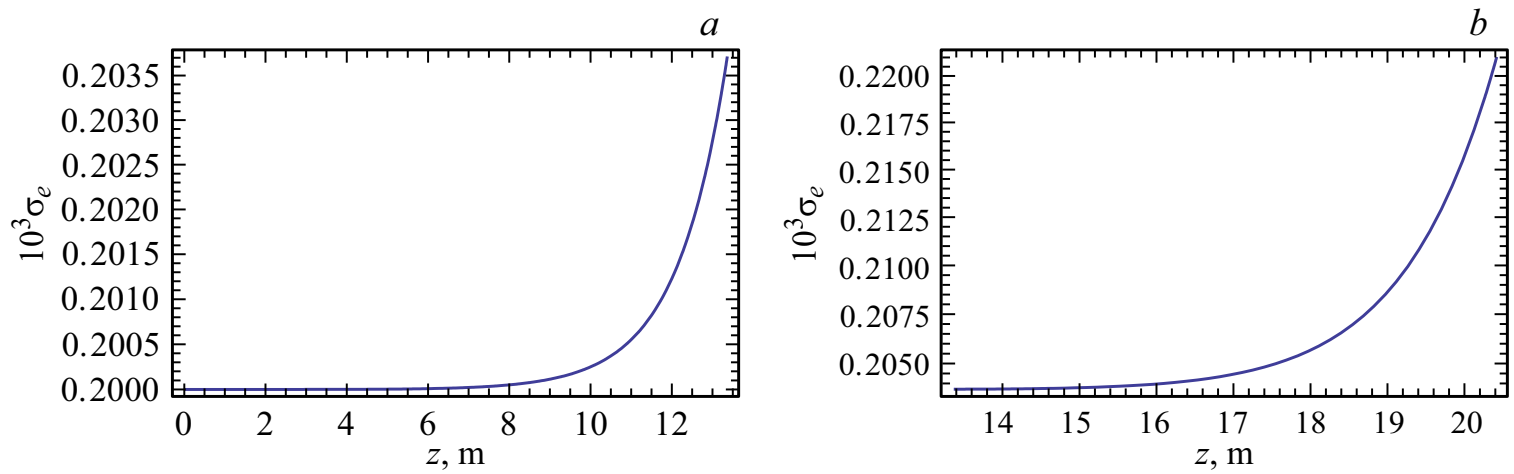

Рис. 4. Эволюция индуцированного ЛСЭ разброса энергии электронов по длине ЛСЭ в группирователе (левый график) и умножителе (правый график); начальный разброс энергии $\sigma_{e}=0.0002$.

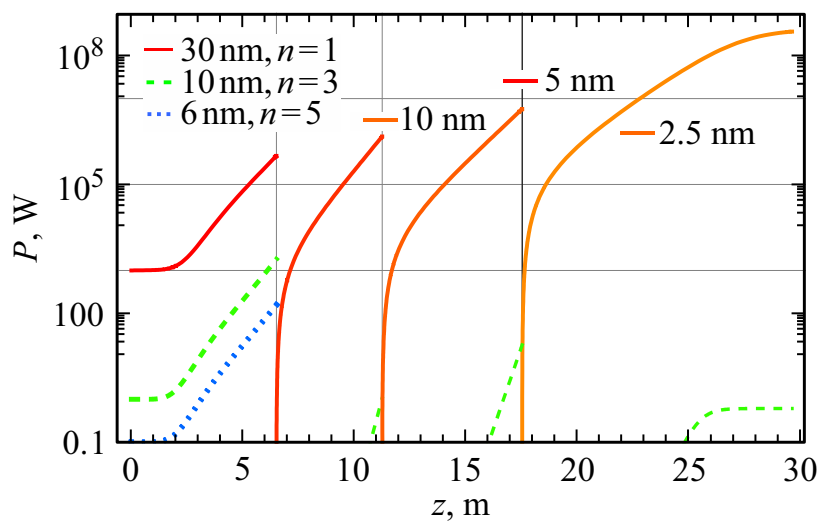

Pис. 5. Эволюция мощности излучения в многокаскадном ЛСЭ с двухчастотным группирователем, затравочным лазером на длине волны $30 \mathrm{~nm}$ и умножителями гармоник; гармоники с $n=1$ - сплошные линии, $n=3$ - пунктирные линии; начальный разброс энергии электронов $\sigma_{e}=0.0001$.

гии. Первый умножитель гармоник настроен на третью гармонику группирователя; все последующие каскады настроены на вторую гармонику предшествующего каскада соответственно. Вторые гармоники в плоском ондуляторе не излучаются; эквивалентная мощность для следующего каскада обеспечивается на их длинах волн банчингом, индуцированным за счет основного тона каскада. Эволюция мощности гармоник в таком ЛСЭ представлена на рис. 5. Основные детали моделирования приведены ниже.

$f_{n, 1}=\{0.756,0.339,0.201\}$,

$f_{n, 2}=\{0.845,0.298,0.153\}$

$f_{n, 3}=\{0.923,0.188,0.055\}$,

$f_{n, 4}=\{0.971,0.080,0.009\}$,

$\rho_{n, 1}=\{0.0018,0.0011,0.0007\}$,

$\rho_{n, 2}=\{0.0010,0.0005,0.0003\}$,

$\rho_{n, 3}=\{0.0006,0.0002,0.00008\}$,

$\rho_{n, 4}=\{0.0003,0.00006,0\}, \quad \gamma=1250, \quad P_{E}=1000 \mathrm{GW}$,

$J=1.0 \cdot 10^{10} \mathrm{~A} / \mathrm{m}^{2}, \quad \Sigma=1.566 \cdot 10^{-7} \mathrm{~m}^{2}, \quad I_{0}=1.56 \mathrm{kA}$,

$\sigma_{e}=0.0001, \quad \xi=1.03, \quad k=2.13306, \quad h=3, \quad d=0.5$, $\lambda_{u, 1}=2.8 \mathrm{~cm}, \quad L_{s}=14 \mathrm{~m}, \quad L_{\text {gain }}=0.8 \mathrm{~m}, \quad k_{2}=1.5655$, $\lambda_{u, 2}=1.4 \mathrm{~cm}, \quad L_{s, 2}=13 \mathrm{~m}, \quad L_{\text {gain }, 2}=0.7 \mathrm{~m}, \quad k_{3}=0.9$, $\lambda_{u, 3}=1.12 \mathrm{~cm}, \quad L_{s, 3}=11 \mathrm{~m}, \quad L_{\text {gain }, 3}=0.9 \mathrm{~m}, \quad k_{4}=0.5$, $\lambda_{u, 4}=0.7 \mathrm{~cm}, L_{s, 4}=7 \mathrm{~m}, L_{\mathrm{gain}, 4}=1.2 \mathrm{~m}, \lambda_{\mathrm{sec} 1}=30 \mathrm{~nm}$, $\lambda_{\mathrm{sec} 2}=10 \mathrm{~nm}, \lambda_{\mathrm{sec} 1} / \lambda_{\mathrm{sec} 2}=3, \lambda_{\mathrm{sec} 3}=5 \mathrm{~nm}, \lambda_{\mathrm{sec} 2} / \lambda_{\mathrm{sec} 3}=2$, $\lambda_{\mathrm{sec} 4}=2.5 \mathrm{~nm}, \lambda_{\mathrm{sec} 3} / \lambda_{\mathrm{sec} 4}=2$.

После группирователя и двух каскадов умножения гармоник следует излучатель, который выдает $400 \mathrm{MW}$ на длине волны $\lambda_{\mathrm{sec} 4}=2.5 \mathrm{~nm}$. Параметр Пирса в короткопериодном излучателе очень мал: $\rho_{n=1, \sec 4}=0.0003$, так что начальный разброс энергии электронов пучка должен быть также малым: $\sigma_{e} \approx 0.0001$. Мы рассчитали эволюцию разброса энергии по длине ЛСЭ с учетом индуцированного ЛСЭ вклада в каждом каскаде, и выбрали точки разрыва так, чтобы оптимизировать работу ЛСЭ с наименьшей возможной длиной при условии $\sigma_{e} \leq \rho_{\text {radiator }} / 2$. С учетом $\sim 20 \%$ потерь эквивалентной мощности между каскадами в ЛСЭ с коэффициентом умножения частоты $12=3 \times 2 \times 2$ и длиной волны $\lambda_{\mathrm{sec} 4}=2.5 \mathrm{~nm}$ мы получили насыщение на $30 \mathrm{~m} \mathrm{c}$ мощностью $400 \mathrm{MW}$ в излучателе длиной $12 \mathrm{~m}$. Возможно использование ондуляторов с более длинным периодом и большим значением параметра $k$ в каждом каскаде; это позволит несколько ослабить требование к разбросу энергии но увеличит полную длину ЛСЭ.

В заключение рассмотрим многокаскадный ЛСЭ с коэффициентом умножения гармоник $48=3 \times 2 \times 2 \times 2 \times 2$, в котором первый умножитель настроен на третью гармонику двухчастотного группирователя электронов и каждый следующий каскад настроен на вторую гармонику предыдущего каскада. Вторые гармоники не излучаются, соответствующая эквивалентная мощность для следующих каскадов происходит за счет банчинга, индуцированного основной гармоникой. В качестве затравочного можно использовать готовый эксимерный $F_{2}$ лазер на $157 \mathrm{~nm}$. Двухчастотный ондулятор обеспечивает хорошую группировку электронов на длине волны третьей гармоники в линейном режиме. В каждом следующем каскаде происходит удвоение частоты. Излучатель-излучатель также настроен на вторую гармонику предыдущего кас- 


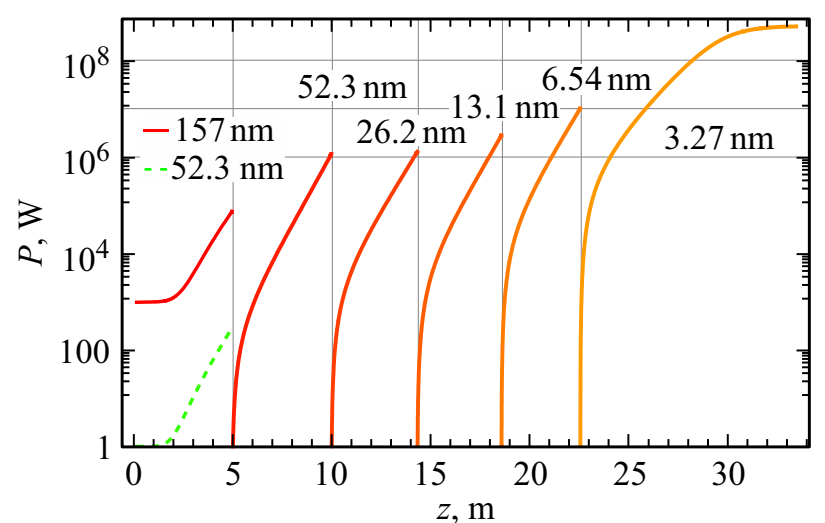

Рис. 6. Эволюция мощности гармоник в многокаскадном ЛСЭ с двухчастотным группирователем, затравочным эксимерным $F_{2}$ лазером на длине волны $157 \mathrm{~nm}$ и умножителями гармоник; начальный разброс энергии электронов $\sigma_{e}=10^{-4}$. Гармоники с $n=1$ - сплошные линии; гармоника с $n=3$ - пунктирные линии.

када, которая усиливается до насыщения и излучается. Основные данные моделирования приведены ниже.

$\rho_{n, 1}=\{0.0038,0.0025,0.0020\}$,

$\rho_{n, 2}=\{0.0023,0.0014,0.0010\}$,

$\rho_{n, 3}=\{0.0017,0.0009,0.0007\}$,

$\rho_{n, 4}=\{0.0011,0.0006,0.0004\}$,

$\rho_{n, 5}=\{0.0007,0.0003,0.0002\}$,

$\rho_{n, 6}=\{0.0004,0.0001,0.00003\}$,

$\gamma=1130, P_{E}=1000 \mathrm{GW}, J=1.0 \cdot 10^{10} \mathrm{~A} / \mathrm{m}^{2}$,

$\Sigma=1.7 \cdot 10^{-7} \mathrm{~m}^{2}, I_{0}=1.73 \mathrm{kA}, \xi=1.05, \sigma_{e}=0.0001$, $k_{1}=3.5, \quad d=0.5, \quad h=3, \quad \lambda_{u, 1}=5.5 \mathrm{~cm}, \quad L_{s}=14 \mathrm{~m}$, $L_{\text {gain }}=0.8 \mathrm{~m}, k_{2}=2.77972, \lambda_{u, 2}=2.75 \mathrm{~cm}, L_{s, 2}=14 \mathrm{~m}$, $L_{\text {gain }, 2}=0.6 \mathrm{~m}, k_{3}=2.01972 \mathrm{~cm}, \lambda_{u, 3}=2.2 \mathrm{~cm}, L_{s, 3}=13 \mathrm{~m}$, $L_{\text {gain, } 3}=0.6 \mathrm{~m}, k_{4}=1.69217, \lambda_{u, 4}=1.375 \mathrm{~cm}, L_{s, 4}=12 \mathrm{~m}$, $L_{\text {gain }, 4}=0.6 \mathrm{~m}, k_{5}=1.28358, \lambda_{u, 5}=0.916667 \mathrm{~cm}$,

$L_{s, 5}=11 \mathrm{~m}, L_{\text {gain }, 5}=0.6 \mathrm{~m}, k_{6}=0.657049$,

$\lambda_{u, 6}=0.6875 \mathrm{~cm}, L_{s, 6}=8 \mathrm{~m}, L_{\text {gain }, 6}=1 \mathrm{~m}$,

$\lambda_{\mathrm{sec} 1}=157 \mathrm{~nm}, \lambda_{\mathrm{sec} 2}=52.4 \mathrm{~nm}, \lambda_{\mathrm{sec} 3}=26.2 \mathrm{~nm}$,

$\lambda_{\mathrm{sec} 4}=13.1 \mathrm{~nm}, \lambda_{\mathrm{sec} 5}=6.55 \mathrm{~nm}, \lambda_{\mathrm{sec} 6}=3.27 \mathrm{~nm}$,

$\lambda_{\mathrm{sec} 1} / \lambda_{\mathrm{sec} 2}=3, \lambda_{\mathrm{sec} 2} / \lambda_{\mathrm{sec} 3}=3, \lambda_{\mathrm{sec} 3} / \lambda_{\mathrm{sec} 4}=2$,

$\lambda_{\sec 4} / \lambda_{\sec 5}=2, \lambda_{\sec 5} / \lambda_{\sec 6}=2$.

Эволюция мощности гармоник ЛСЭ показана на рис. 6. Точки разрыва и длины каскадов тщательно подобраны для минимизации всей длины ЛСЭ с учетом индуцированного в каждом каскаде разброса энергии; расчет выполнен с помощью формулы (10) для каждого каскада. Графики разброса энергии в каждом каскаде не приводятся для краткости. Основные гармоники обозначены сплошными линиями, третья гармоника в группирователе обозначена пунктирной линией на рис. 6 . Высшие гармоники в умножителях индуцируются основными гармониками и растут в нелинейном режиме. Короткопериодный излучатель в последнем каскаде имеет параметры, близкие к заявленным на установке Сибирь 2 в [54]. Возможен выбор более слабых ондуляторов для каскадов; это увеличит общую длину установки. В смоделированном нами многокаскадном ЛСЭ с затравочным $F_{2}$ эксимерным UV-лазером на длине волны $157 \mathrm{~nm}$ используем электронный пучок энергии $E \approx 577 \mathrm{MeV}$ с разбросом энергии $\sigma_{e}=0.0001$. Существенно больший начальный разброс с учетом индуцированного ЛСЭ вклада не удовлетворяет необходимому условию $\sigma_{e} \leq \rho_{\text {radiator }} / 2=0.00021$. После $\sim 30 \mathrm{~m} \mathrm{в} \mathrm{таком} \mathrm{ЛСЭ}$ имеем мощность насыщения $\approx 500 \mathrm{MW}$ на длине волны мягкого рентгеновского излучения $\lambda \approx 3.27 \mathrm{~nm}$. Более того, за счет высококачественного готового затравочного лазера излучение ЛСЭ получает не только хорошие пространственные моды, характерные для ЛСЭ с ССИ, но и хорошую временную когерентность, присущую оптическим лазерам.

Отметим, что из рассмотренных выше примеров следует, что при значительном разбросе энергий электронов, короткой длине волны излучения и большом коэффициенте умножения ЛСЭ, высшие гармоники оказываются сильно подавлены и предпочтительно использование гармоник с $n=2$ с большим числом умножающих каскадов, в отличие от каскадных ЛСЭ в [55-59], где использовались третья и пятая гармоники.

\section{Результаты и выводы}

При помощи феноменологической модели с учетом основных потерь мы рассмотрели несколько однопроходных каскадных ЛСЭ с УВГ и с умножением гармоник, и проследили эволюцию мощности излучения по длине. Разделение ЛСЭ на каскады оптимизировано для получения наиболее мощного рентгеновского излучения на минимальной длине установки. Моделирование проводилось в программе Mathematica. Нами предложены каскадные ЛСЭ для генерации мягкого рентгеновского излучения длиной волны несколько нанометров с затравочным излучением на длине волны отражения $\mathrm{Mo} / \mathrm{Si}$, $\lambda_{\text {seed }}=13.51 \mathrm{~nm}$, и $\mathrm{MoRu} / \mathrm{Be}, \lambda_{\text {seed }}=11.43 \mathrm{~nm}$, а также с затравочным UV-лазером на длине волны $\lambda_{\text {seed }}=157 \mathrm{~nm}$ и гармоникой лазера с $\mathrm{CO}_{2}$ или с $\mathrm{C}_{2} \mathrm{H}_{2}$ на длине волны $\lambda_{\text {seed }}=30 \mathrm{~nm}$. В каскаде группирователя электронов предпочтительно использование двухчастотных плоских ондуляторов, которые дают значительно большее усиление гармоник в линейном режиме, чем обычные плоские ондуляторы. Это позволяет осуществлять группировку электронов непосредственно на длинах волн гармоник с минимальным индуцированным ЛСЭ разбросом энергий. Работа каскадных ЛСЭ с умножением гармоник исследовалась при разбросе энергий пучка электронов в диапазоне $\sigma_{e}=0.00005-0.0005$; большие значения $\sigma_{e}$ неприемлемы для каскадных ЛСЭ в рентгеновском диапазоне. Мощность высших гармоник сильно падает с ростом разброса энергий электронов. При $\sigma_{e}>0.00015$ требуются излучатели существенно большей длины, чем при $\sigma_{e}<0.0001$. 
Таким образом, предпочтительным для каскадных ЛСЭ с умножением гармоник в рентгеновском диапазоне является использование пучков с разбросом энергии $\sigma_{e} \approx 0.0001$. При $\sigma_{e} \leq 0.0002$ возможно использование двухчастотных ондуляторов для генерации высших гармоник в группирователе; при $\sigma_{e}>0.0003$ группировка на длинах волн гармоник становится очень слабой. Для обычных плоских ондуляторов это происходит уже при $\sigma_{e}>0.0001$. Двухчастотные ондуляторы лучше группируют электроны на высших гармониках.

Исследована эволюция мощности гармоник ЛСЭ с УВГ с электронами энергии $E=950 \mathrm{MeV}$, разбросом энергий $\sigma_{e}=0.0001$ и током $I_{0} \sim 100 \mathrm{~A}$; двухчастотный ондулятор-группирователь имеет период $\lambda_{u, 1}=2.8 \mathrm{~cm}$, $k=2.133, h=3$ и $d=0.5$, излучатель имеет период $\lambda_{u, 2}=1.25 \mathrm{~cm}$ и $k_{2}=1$, затравочное излучение на длине волны $\lambda_{\text {seed }}=13.51 \mathrm{~nm}$ соответствует пику отражения $\mathrm{Mo} / \mathrm{Si}$. В результате получаем излучение на длине волны $\lambda_{\sec 2}=2.7 \mathrm{~nm}$ мощностью $55 \mathrm{MW}$ на длине ЛСЭ $40 \mathrm{~m}$ и третью гармонику на длине волны $\lambda_{\sec 3, n=3}=0.9 \mathrm{~nm}$ с мощностью $40 \mathrm{~kW}$ после $35 \mathrm{~m}$ ЛСЭ. При разбросе энергий $\sigma_{e}=0.00025$ требуемая длина дорогого короткопериодного излучателя ЛСЭ существенно возрастает по сравнению с его длиной при $\sigma_{e} \approx 0.0001$. С током $I_{0} \sim 1 \mathrm{kA}$ получаем $\approx 0.6 \mathrm{GW}$ излучения на длине волны основного тона ЛСЭ $\lambda_{\sec 2}=2.71 \mathrm{~nm}$ с длиной насыщения $\approx 45 \mathrm{~m}$ (рис. 1 ).

Для генерации когерентного рентгеновского излучения с длиной волны $\lambda_{\sec 3}=2.25 \mathrm{~nm}$ предлагается затравочный лазер на длине волны $\lambda_{\text {seed }}=13.51 \mathrm{~nm}$ пика отражения $\mathrm{Mo} / \mathrm{Si}$, и электроны энергии $E=950 \mathrm{MeV}$ с током $I_{0} \sim 1 \mathrm{kA}$ и разбросом энергии $\sigma_{e}=0.0002$. При этом предложен каскад умножения гармоник на два, что позволяет генерировать $\sim 0.5 \mathrm{GW}$ излучения уже на $\approx 35 \mathrm{~m}$ ЛСЭ, что короче, чем в ЛСЭ с УВГ с разбросом энергии $\sigma_{e}=0.0001$ (ср. рис. 3 с рис. 1). При этом длина излучателя в каскадном ЛСЭ оказывается на $\approx 10 \mathrm{~m}$ короче, чем в ЛСЭ с УВГ.

В многокаскадном ЛСЭ с пучком электронов энергии $E \approx 640 \mathrm{MeV}$, разбросом энергии $\sigma_{e}=0.0001$, током $I_{0} \approx 1.56 \mathrm{kA}$ и затравочным излучением гармоники лазера с $\mathrm{CO}_{2}, \lambda_{\text {seed }}=30 \mathrm{~nm}$, можно получить $\approx 400 \mathrm{MW}$ излучения с длиной волны $\lambda_{\mathrm{sec} 4}=2.5 \mathrm{~nm}$ уже на $\approx 30 \mathrm{~m}$ ЛСЭ (рис.5).

Исследована генерация гармоник в ЛСЭ с готовым $F_{2}$ эксимерным UV-лазером на $\lambda_{\text {seed }}=157 \mathrm{~nm}$. Показано, что с электронами энергии $E=577 \mathrm{MeV}$, разбросом энергий $\sigma_{e}=1 \cdot 10^{-4}$ и током $I_{0} \approx 1.73 \mathrm{kA}$ можно получить $\sim 0.5 \mathrm{GW}$ мягкого рентгеновского излучения на длине волны $\lambda_{\sec 5}=3.27 \mathrm{~nm}$ в ЛСЭ с ондуляторами длиной $\sim 30 \mathrm{~m}$. Использование в качестве затравочного излучения готового UV лазера значительно упрощает и удешевляет конструкцию рентгеновского ЛСЭ. Двухчастотный ондулятор обеспечивает эффективную группировку электронов непосредственно на длинах волн его гармоник, а следующие каскады производят умножение частоты в 48 раз.
Таким образом, используя двухчастотные ондуляторыгруппирователи электронов и каскадные умножители гармоник в ЛСЭ, с электронами энергии $E \sim 0.5-1 \mathrm{GeV}$, разбросом энергии около $\sigma_{e} \approx 10^{-4}$ и током $I_{0} \approx 1 \mathrm{kA}$ можно получить до 1 ГВТ мягкого рентгеновского излучения на длине волны около нанометра уже на $\sim 30-40 \mathrm{~m}$. Излучение таких ЛСЭ будет иметь стабильную фазу, высокую пространственную и временную когерентность за счёт использования затравочного излучения со стабильной фазой. Для этого можно использовать резонаторы из $\mathrm{MoRu} / \mathrm{Be}, \mathrm{Mo} / \mathrm{Si}$, или готовые эксимерные UV лазеры и их гармоники. Стоимость и размер предложенных ЛСЭ для генерации мягкого рентгеновского излучения значительно меньше, чем гигантских установок типа X-FEL и др.

Авторы благодарят проф. А.В. Борисова, проф. В.Ч. Жуковского, проф. А.Н. Васильева, проф. А.Е. Лобанова и А.М. Калитенко за полезные обсуждения и замечания.

\section{Список литературы}

[1] Багров В.Г., Бисноватый-Коган Г.С., Бордовищын В.А. Теория излучения релятивистских частиц. М.: Физматлит, 2002. $575 \mathrm{c}$.

[2] Багров В.Г., Тернов И.М., Холомай Б.В. Излучение релятивистских электронов в продольном периодическом электрическом поле кристалла. Томск: ТФ СО АН СССР, 1987. $13 \mathrm{c}$.

[3] Motz H., Thon W., Whitehurst R.N.J. // Appl. Phys. 1954. Vol. 24. P. 826.

[4] Гинзбург В.Л. // Изв. АН СССР (Физика). 1947. Т. 11. C. 1651.

[5] Zastrau U., Burian T., Chalupsky J. // Laser Part Beams. 2012. Vol. 30. P. 45.

[6] Ziaja B., Weckert E., Möller T. // Laser Part. Beams. 2007. Vol. 25. P. 407.

[7] Lee R. W., Baldis H. A., Cauble R.C. // Laser Part Beams. 2002. Vol. 20. P. 527.

[8] Kumar S., Kang H.-S., Kim D.-E. // Laser Part Beams 2012. Vol. 30. P. 397.

[9] Bartnik A. et al. // Laser Part Beams. 2013. Vol. 31. P. 195.

[10] Huang H., Tallents G. J. // Laser Part Beams Vol. 2009. Vol. 27. P. 393.

[11] McNeil B.W.J., Thompson N.R. // Nature Photonics. 2010. Vol. 4. P. 814.

[12] Pellegrini C., Marinelli A., Reiche S. // Mod. Phys. 2016. Vol. 88. P. 015006.

[13] Huang Z., Kim K.J. // Phys. Rev. ST-AB. 2007. Vol. 10. P. 034801.

[14] Saldin E.L., Schneidmiller E.A., Yurkov M.V. The Physics of Free Electron Lasers. Springer, 2000.

[15] Schmüser P., Dohlus M., Rossbach J., Behrens C. FreeElectron Lasers in the Ultraviolet and X-Ray Regime, Springer Tracts in Modern Physics. Springer, 2014. 258 c.

[16] Emma P. et al. // Nature Photonics. 2010. Vol. 4. P. 641.

[17] Artyukov I.A., Bessonov E.G., Gorbunkov M.V. // Laser Part. Beams. 2016. Vol. 34. P. 637.

[18] Bessonov E.G. et al. // Laser Part. Beams 2008. Vol. 26. P. 489. 
[19] Frank M. et al. // IUCrJ. 2014. Vol. 1. P. 95.

[20] Бессонов Е.Г. // Квантовая электроника. 1986. Vol. 13. C. $1617-1628$.

[21] Белявский Е.Д., Теличкина О.В. // ЖТФ. 2013. Т. 83. Вып. 4. С. 123-126.

[22] Bessonov E.G. // Nucl. Instr. Meth. A. 1989. Vol. 282. P. 442444.

[23] Altarelli M. et al. European XFEL Annual Report. 2016. XFEL.EUAR-2016. DOI: 10.22003/XFEL.EU-AR-2016

[24] Yu L.-H. et al. // Science. 2000. Vol. 289. P. 932.

[25] Shaftan T., Yu L.-H. // Phys. Rev. E. 2005. Vol. 71. P. 046501.

[26] Zhukovsky K.V. // Moscow Univ. Phys. Bull. 2015. Vol. 70. P. 232.

[27] Zhukovsky K. // J. Electromagn. Wave. 2015. Vol. 29. P. 132.

[28] Zhukovsky K. // J. Electromagn. Wave. 2014. Vol. 28. P. 1869.

[29] Mishra G., Gehlot M., Hussain J.-K. // Nucl. Instrum. A. 2009. Vol. 603. P. 495.

[30] Dattoli G., Mikhailin V. V., Ottaviani P. L., Zhukovsky K. // J. Appl. Phys. 2006. Vol. 100. P. 084507.

[31] Zhukovsky K. // Laser Part. Beams. 2016. Vol. 34. P. 447.

[32] Dattoli G., Mirian N.S., DiPalma E., Petrillo V. // Phys. Rev. ST-AB. 2014. Vol. 17. P. 050702.

[33] Shintake T. // Nature Photonics. 2008. Vol. 2. P. 555-559. DOI: $10.1038 /$ nphoton.2008.134

[34] McNeil B. // Nature Photonics. 2008. Vol. 2. P. 522-524. DOI: $10.1038 /$ nphoton.2008.164

[35] Tiedtke K. et al. // New J. Phys. 2009. Vol. 11. P. 023029.

[36] Madey J.M.J. // J. Appl. Phys. 1971. Vol. 42. P. 1906.

[37] Dattoli G., Ottaviani P.L., Renieri A. // Laser Part. Beams. 2005. Vol. 23. P. 303.

[38] Dattoli G., Ottaviani P.L., Pagnutti S. // J. Appl. Phys. 2005. Vol. 97. P. 113102.

[39] Dattoli G., Giannessi L., Ottaviani P.L., Ronsivalle C. // J. Appl. Phys. 2004. Vol. 95. P. 3206-3210.

[40] Zhukovsky K., Potapov I. // Laser Part. Beams. 2017. Vol. 35. P. 326.

[41] Giannessi L. et al. // Phys. Rev. ST-AB, 2011. Vol. 14. P. 060712.

[42] Zhukovsky K. // Nucl. Instrum. Meth. Phys. Res. B. 2016. Vol. 369. P. 9.

[43] Zhukovsky K. // Opt. Commun. 2015. Vol. 353. P. 35.

[44] Quattromini M., Artioli M., Di Palma E., Petralia A., Giannessi L. // Phys. Rev. ST-AB, 2012. Vol. 15. P. 080704.

[45] Dattoli G., Ottaviani P.L., Pagnutti S. Booklet for FEL design. Frascati, Italy: ENEA Pubblicazioni, 2007.

[46] Walker R.P. // Nucl. Instrum. Method. A. 1993. Vol. 335. P. 328-337.

[47] Грейсух Г.И., Ежсов Е.Г., Казин С.В., Степанов С.А. // ЖТФ. 2012. Т. 82. С. 99-103.

[48] Onuki H., Elleaume P. Undulators, wigglers and their applications. NY:: Taylor \& Francis, 2003.

[49] Zhukovsky K. // J. Optics. 2018. Vol. 20. N 9. P. 095003.

[50] Patent numbers: EP $1198725 \mathrm{~A} 1$ and PCT number PCT/US2000/013549

[51] Zhukovsky K.V. // Moscow Univ. Phys. Bull. 2017. Vol. 72. P. 128.

[52] Ling Zeng et al. // Chinese Phys. C. 2016. Vol. 40. P. 098102.

[53] Sayrac M., Kolomenskii A.A., Schuessler H.A. // J. Electron Spectrosc. Relat. Phenom. 2018. Vol. 29. P. 1-6.

[54] Корчуганов В.Н., Свечников Н.Ю., Смоляков Н.В., Томин С.И. // Поверхность. 2010. Т. 11. С. 22-28.
[55] Zhukovsky K.V. // Russian Phys. J. 2018. Vol. 60. N 9. P. 1630-1637.

[56] Zhukovsky K. // EPL. 2017. Vol. 119. P. 34002.

[57] Zhukovsky K. // J. Phys. D. 2017. Vol. 50. P. 505601.

[58] Zhukovsky K. // Opt. Comm. 2018. Vol. 418. P. 57-64.

[59] Zhukovsky K. // J. Appl. Phys. 2017. Vol. 122. P. 233103. 